\title{
Transfer Learning with Graph Neural Networks for Optoelectronic Properties of Conjugated Oligomers
}

\author{
Chee-Kong Lee, ${ }^{1}$ Chengqiang Lu, ${ }^{2}$ Yue Yu, ${ }^{3}$ Qiming Sun, ${ }^{1}$ Chang-Yu Hsieh, ${ }^{4}$ Shengyu Zhang, ${ }^{4}$ Qi Liu, ${ }^{2}$ and \\ Liang Shi ${ }^{3}$, a) \\ 1) Tencent America, Palo Alto, CA 94306, United States \\ ${ }^{2)}$ Anhui Province Key Lab of Big Data Analysis and Application, University of Science and Technology of China, \\ Hefei, Anhui 230026, China \\ 3) Chemistry and Chemical Biology, University of California, Merced, California 95343, \\ United States \\ 4) Tencent, Shenzhen, Guangdong 518057, China
}

(Dated: 14 November 2020)

Despite the remarkable progress of machine learning (ML) techniques in chemistry, modeling the optoelectronic properties of long conjugated oligomers and polymers with ML remains challenging due to the difficulty in obtaining sufficient training data. Here we use transfer learning to address the data scarcity issue by pretraining graph neural networks using data from short oligomers. With only a few hundred training data, we are able to achieve an average error of about $0.1 \mathrm{eV}$ for excited state energy of oligothiophenes against TDDFT calculations. We show that the success of our transfer learning approach relies on the relative locality of low-lying electronic excitations in long conjugated oligomers. Finally, we demonstrate the transferability of our approach by modeling the lowest-lying excited-state energies of poly(3-hexylthiopnene) (P3HT) in its single-crystal and solution phases using the transfer learning models trained with data of gas-phase oligothiophenes. The transfer learning predicted excited-state energy distributions agree quantitatively with TDDFT calculations and capture some important qualitative features observed in experimental absorption spectra.

\section{INTRODUCTION}

Conjugated oligomers and polymers are semiconducting organic molecules with extended $\pi$-conjugated backbones, over which $\pi$ electrons can be delocalized. Due to their desirable electronic and photophysical properties, they have found wide applications in many fields, spanning from optoelectronics ${ }^{1-3}$ to bio-sensing and bioimaging. ${ }^{4}$ For these applications, it is critical to understand the relationship between the microscopic structures and electronic properties of these materials. However, predicting their electronic properties is a challenging task mostly for two reasons: quantum chemical calculations for long oligomers and polymers are very expensive, if not impossible, even with density functional theory (DFT); and their electronic properties are very sensitive to their conformations, which are often highly heterogeneous at ambient temperature. To address the first issue, a common strategy is to infer the electronic properties of long oligomers/polymers by extrapolating short oligomer properties, and empirical relations have been devised. ${ }^{5-9}$ However, this approach is mostly used with optimized geometries, and how to learn from short oligomers to predict the properties of long oligomers/polymers at their nonequilibrium geometries remains largely unexplored. In this work, following the extrapolation idea we advocate the use of transfer learning, a machine learning (ML) technique, to tackle this problem.

ML has attracted significant interests from the scientific community due to its potential to accelerate atom-

\footnotetext{
a) Electronic mail: lshi4@ucmerced.edu
}

istic calculations by orders of magnitude. ${ }^{10-15}$ Specifically for organic semiconductors, a plethora of ML methods, such as support vector machine (SVM), kernel ridge regression (KRR), feed-forward neural network (NN), and random forests $(\mathrm{RF})$, have been applied to study their electronic properties for various (opto)electronic applications. ${ }^{16-41}$ The success of ML methods in many of these studies relied on careful feature selection, while recent graph-based ML approaches, such as SchNet $^{42}$ and Message Passing Neural Networks (MPNN), ${ }^{43}$ are capable of extracting the optimal representation of a molecule solely from atom types and Cartesian coordinates. These state-of-the-art ML methods have shown impressive performance on benchmark datasets such as the QM9 dataset which contains the ground state properties of molecules consisting of up to 9 non-hydrogen atoms. ${ }^{42-55}$ Despite the remarkable progress of these graph ML methods, their application to conjugated long oligomers and polymers remains limited primarily due to the difficulty in obtaining sufficient training data using quantum chemical methods.

A popular technique in ML to address data scarcity is transfer learning, in which an ML model is trained on data from one task, then the model is re-used as the starting point for a different but related task where training data is often limited. ${ }^{56,57}$ There were already several applications of transfer learning in chemistry, and these include transferring models trained on DFT data to $\operatorname{CCSD}(\mathrm{T}) / \mathrm{CBS}$ data: ${ }^{58}$ applying models pre-trained on small molecules to larger ones (up to 14 non-hydrogen atoms) $;^{59}$ development of pretrained library XenonPy.MDL from publicly available datasets $;{ }^{60}$ using pre-trained models for QSPR/QSAR 
predictions; ${ }^{61,62}$ virtual screening of donor organic photovoltaic molecules ${ }^{63}$ and solid lithium-ion conductors; ${ }^{64}$ and autoencoder approach to transfer learning. ${ }^{65,66}$

In this work, we apply transfer learning to predict the electronic properties of long oligomers using training data on short oligomers. Using oligothiophenes (OTs) as an example (see Fig. 1), we first pre-train a graph neural network (GNN) with data from short oligomers of various lengths whose repeating unit is the same as that of the target long oligomers. Since computing electronic properties of short oligomers with quantum chemistry is very affordable, it is relatively easy to generate a training dataset comprising a large number of (nonequilibrium) configurations of short oligomers. After pre-training, the GNN model is then fine-tuned with a small number of training data of the target long oligomer. We find that the use of pre-training could improves the accuracy of GNNs by as much as $37 \%$ compared to the results without transfer learning. The rest of the paper is organized as follows. In Sec. II, we provide the details of training data generation and model training. In Sec. III, we assess the performance of the transfer learning models in predicting the HOMO-LUMO gap and excitation energy of long OTs, provide a physical explanation for their success, and demonstrate their transferability by applying them to a substituted polythiophene in its condensed phases. In Sec. IV, we conclude.

\section{COMPUTATIONAL DETAILS}

$\alpha$-linked OTs are chosen as our systems to demonstrate the effectiveness of transfer learning, and they are denoted as $n \mathrm{~T}$, where $n$ is the number of thiophene rings in the OT. OTs have been extensively studied both experimentally and theoretically due to their optoelectronic properties and their relevance to polythiophenes and their derivatives, such as poly(3hexylthiopnene) (P3HT), a widely used organic semiconducting polymer. ${ }^{67,68}$ There are many studies suggesting that the electronic properties of polythiophenes (PTs) may be inferred from those of OTs, and the estimated effective conjugation length of unsubstituted or substituted PTs varies greatly, ranging from about 11 to 96 thiophene units. ${ }^{6,69-75}$ Empirical expressions have also been devised to correlate the excitation energy and the size of OTs and PTs, ${ }^{6,73-75}$ and despite their differences, the difference in excitation energy between 10T and the infinite long $\mathrm{nT}$ predicted by these empirical relationships is about $300 \mathrm{meV}$. Considering the computational affordability and the typical errors of time-dependent density functional theory (TDDFT) in estimating singlet excitation energy (i.e., 100-500 meV), we choose $2 \mathrm{~T}-10 \mathrm{~T}$ as the short OTs in the pre-training phase, and the target properties for transfer learning are the excited-state energies and HOMO-LUMO gaps of 11T-16T.

\section{A. Data Generation}

To adequately sample the configuration space of OTs and test the performance of transfer learning over a wide range of configurations, classical molecular dynamics (MD) simulations were performed for each OT in its gas phase at $1000 \mathrm{~K}$. Sufficiently large simulation boxes were employed with periodic boundary condition. Simulations were performed in the NVT ensemble at $1000 \mathrm{~K}$ with the Nosé-Hoover thermostat ${ }^{76,77}$ and a coupling constant of $2.0 \mathrm{ps}$, and the particle-mesh Ewald $\operatorname{method}^{78,79}$ was used for electrostatic interactions. All the MD simulations were performed with the OPLS/2005 force field ${ }^{80}$ in the Desmond package 3.6. ${ }^{81}$ OPLS/2005 force field ${ }^{80}$ was adopted for its capability of reproducing the torsional potential energy surface of (unsubstituted) bithiophene from localized second-order Møller-Plesset perturbation theory. ${ }^{82}$ The simulation time step was $1 \mathrm{fs}$, and during the production run configurations were saved every 100 fs over a 2-ns trajectory for each OT. Configurations were randomly drawn from the trajectories for subsequent quantum chemical calculations.

DFT was employed to compute the HOMO-LUMO gap, and TDDFT with the Tamm-Dancoff approximation was used for the excitation energy. CAM-B3LYP functional and 6-31+G(d) basis set were chosen based on the agreement with coupled cluster calculations in our previous study, ${ }^{40}$ where the average error for the excitation energies of OTs up to 6T using CAM-B3LYP/6$31+\mathrm{G}(\mathrm{d})$ was estimated to be around $200-300 \mathrm{meV}$. All the quantum chemical calculations were performed using the PySCF program, ${ }^{83}$ and density fitting was adopted with the heavy-aug-cc-pvdz-jkfit auxiliary basis set, as implemented in PySCF.

\section{B. Model Training}

The specific GNN model we choose for our transfer learning protocol is SchNet since it has been shown in our previous study to provide the best performance for a range of ground and excited state properties of OTs up to $6 \mathrm{~T} .{ }^{40}$ Similar to other GNN models, SchNet is capable of automatically extracting optimal representations from molecular configurations without resorting to the more traditional approach of manually designing descriptors such as Coulomb matrices, ${ }^{84,85}$ bags of bonds, ${ }^{86}$ smooth overlap of atomic positions ${ }^{87}$ or generalized symmetry functions. ${ }^{88}$ In our previous work, we have shown that SchNet can reliably predict various electronic properties (e.g., HOMO-LUMO gap and excited-state energies) of OTs up to $6 \mathrm{~T}$ with the average errors in the range of $20-80 \mathrm{meV} .{ }^{40}$ It is worth emphasizing that the transfer learning protocol advocated here does not depend on the specific underlying ML model, a similar performance improvement could be obtained even when a different ML model is used, as demonstrated in the Supporting Information (SI) where we use a multilevel graph convo- 
(a)

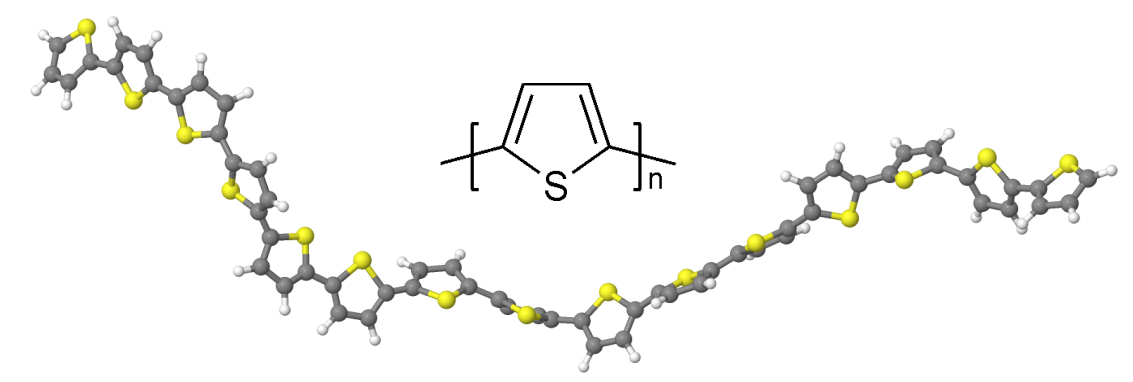

(b)
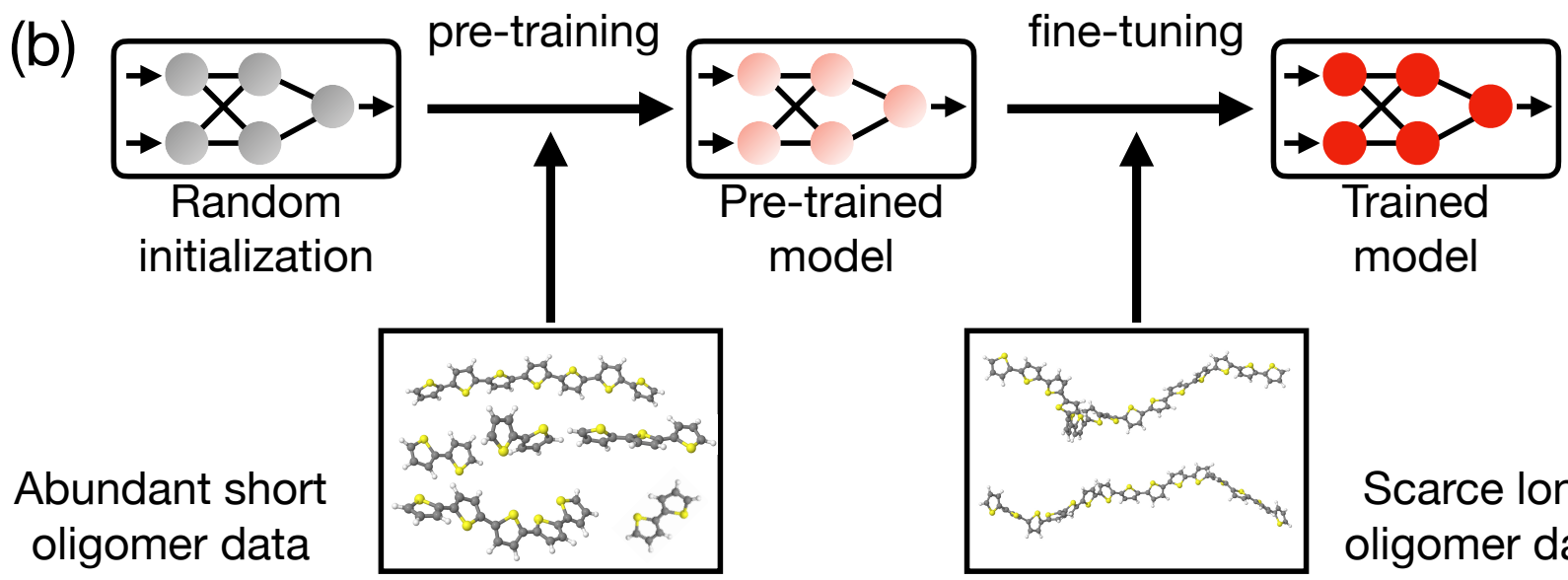

Scarce long
oligomer data

FIG. 1. (a) We use oligothiophenes (OTs) of varying lengths to demonstrate the transfer learning protocol. A sample configuration of an OT molecule (i.e., 15T) is shown. (b) Schematic of transfer learning protocol used in this work. The weights of a GNN model are first randomly initialized. Subsequently, the GNN model is pre-trained using data of short oligomers that resemble the target molecule. Training data of these short oligomers can be easily obtained through electronic structure calculations. After pre-training, the model is then fine-tuned with data of the target long oligomer. The amount of data of the target long oligomer is typically limited.

lutional neural network (MGCN) as the underlying ML model. $^{48}$

The transfer learning protocol used in this work is outlined in Fig. 1(b). SchNet is first pre-trained using data from 2T-10T (1000 for each OT, and 9000 in total) for 300 epochs, the weights of SchNet are then fine-tuned for up to 100 epochs using data from the target molecule (i.e., 11T-16T). We use the same hyperparameters as the original SchNet paper, ${ }^{42}$ unless otherwise stated. Particularly, a cutoff of $5 \AA$ is used for the predictions of all electronic properties. The dimension of embedding in SchNet is set to be 128, and we use three interaction blocks. We train SchNet with Adam optimizer ${ }^{89}$ using a learning rate of 0.0001 and a batch size of 64 . The decay rate is set to 1 (i.e., no decay) since we find that adding decay does not lead to noticeable improvement. In the final training phase, only 200 training data of the target molecules are used, unless otherwise stated (e.g., in Fig. 3). Additionally, 500 validation data are used for early stopping in the final training phase, and the trained models are evaluated using 1000 test data. To demonstrate the performance improvement offered by the transfer learning, we also train SchNet models without transfer learning for up to 400 epochs, and again use the validation set for early-stopping. Our version of SchNet is im- plemented using PyTorch ${ }^{90}$ and Deep Graph Library, ${ }^{91}$ and the source codes can be found on GitHub. ${ }^{92}$

We note that using less validation data for early stopping could lead to over-fitting of the models and consequently poor performance on test data. In case that generating the additional validation data is not feasible, we recommend that one can simply train SchNet with the training data of the target molecules for 100 epochs without early stopping. We found that the errors of such approach are on average about $7 \%$ higher than those with early stopping, still a significant improvement over results without transfer learning

\section{RESULTS AND DISCUSSIONS}

\section{A. Model Performance}

The mean average errors (MAEs) of the first excitedstate energies and HOMO-LUMO gaps of 11T-16T from our transfer learning protocol are shown in Fig. 2. Without transfer learning, the MAEs of the excited state energy range from $170 \mathrm{meV}$ to $187 \mathrm{meV}$ (solid blue line) for $11 \mathrm{~T}$ to $16 \mathrm{~T}$. These results from direct training are already notable as compared to other traditional ML methods 
(a)

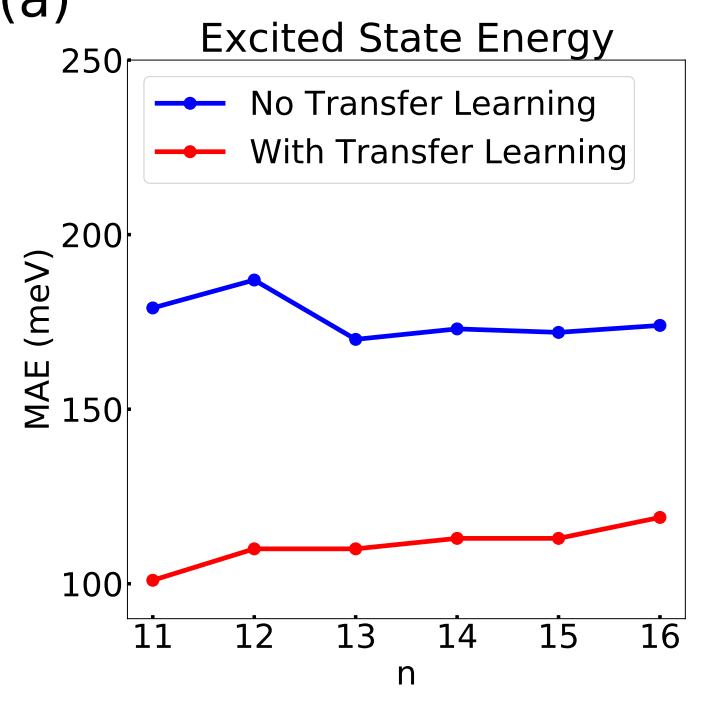

(b)

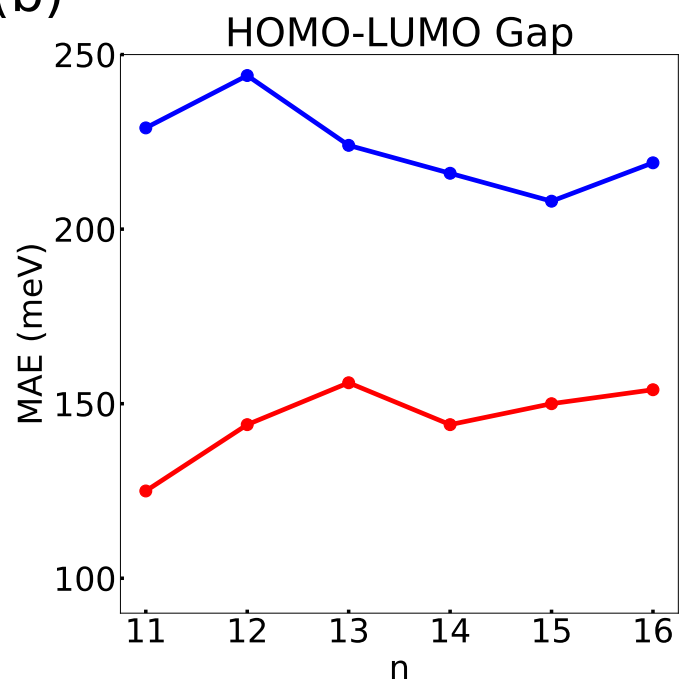

FIG. 2. Mean average errors (MAEs) of (a) excited state energy and (b) HOMO-LUMO gap of OTs as a function of molecular size where $n$ denotes the number of thiophene rings in the molecules. Blue lines represent the results from direct training of SchNet without transfer learning, whereas the red lines denote the results of SchNet pre-trained with $2 \mathrm{~T}-10 \mathrm{~T}$ data. 200 training data of the target long OT are used in all the simulations.

such as shallow feed-forward neural network whose MAEs could exceed $400 \mathrm{meV}$ even with 5 times as many training data (i.e., 1000 training data) for molecules as small as $6 \mathrm{~T} .{ }^{40}$ The performance of SchNet is further boosted with the use of transfer learning: the corresponding MAEs reduce to $101-119 \mathrm{meV}$, an average improvement of $37 \%$ or $65 \mathrm{meV}$. Given our transfer learning protocol, one might anticipate that the errors might increase with the size of OTs as the target long OTs become increasingly different from the small OTs used in the pre-training. Interestingly, we find that the increase in MAEs is fairly minuscule, implying the robustness of our transfer learning method at least up to $16 \mathrm{~T}$.

Similar to the results on excited-state energy, the MAEs of HOMO-LUMO gap range from $208 \mathrm{meV}$ to $244 \mathrm{meV}$ without transfer learning, and transfer learning reduces the MAEs to $125-156 \mathrm{meV}$, with an average improvement of $36 \%$ or $78 \mathrm{meV}$. The MAEs of HOMOLUMO gap are larger than those of excited state energy because its distribution is broader. For example, the standard deviation of $15 \mathrm{~T}$ HOMO-LUMO gap in the data is $322 \mathrm{meV}$ whereas that of excited state energy is 246 $\mathrm{meV}$. The larger standard deviation of HOMO-LUMO gap is likely due to the absence of exciton binding energy which makes the absolute value of HOMO-LUMO gap larger than that of excited state energy. For example, the average value of $15 \mathrm{~T}$ HOMO-LUMO gap is $5.1 \mathrm{eV}$ whereas that of excited state energy is $3.1 \mathrm{eV}$.

Next we explore how the performance of transfer learning depends on the amount of training data in Fig. 3, which shows the MAEs of $15 \mathrm{~T}$ excited state energy (blue lines) and HOMO-LUMO gap (red lines) as functions of $15 \mathrm{~T}$ training data (panel (a)) and as functions of pretraining data from each of the 2T-10T molecules (panel (b)). From Fig. 3 (a), it can be seen that without any data from the target molecule 15T, the MAEs for excited state energy and HOMO-LUMO gap are $279 \mathrm{meV}$ and $623 \mathrm{meV}$, respectively. However, adding as few as 100 training data from the target molecule leads to significant improvement in performance and reduces the MAEs to $114 \mathrm{meV}$ for excited state energy and $155 \mathrm{meV}$ for HUMO-LUMO gap. Further increase of training data only marginally improves the performance of SchNet. For example, with $500 \mathrm{~T} 15$ data, the MAEs reduce to $98 \mathrm{meV}$ for excited state energy and $132 \mathrm{meV}$ for HOMO-LUMO gap. This represents approximately $15 \%$ reduction of MAE even with five times as many training data.

It is also instructive to learn how the performance of transfer learning depends on the amount of data used in the pre-training phase, as depicted in Fig.3 (b). The xaxis of Fig.3 (b) denotes the number of data from each of $2 \mathrm{~T}-10 \mathrm{~T}$ used in the pre-training phase, while the number of $15 \mathrm{~T}$ training data is fixed at 200. The MAE of excitedstate energy decreases from $162 \mathrm{meV}$ to $113 \mathrm{meV}$ as we increase the amount of pre-training data from 0 to 1000 per short OT. Correspondingly, the MAEs for HOMOLUMO gap drops from $208 \mathrm{meV}$ to $150 \mathrm{meV}$ within the same range of pre-training data. Even though our MAEs have not yet reached convergence with respect to the size of the pre-training dataset, it is clear that expanding the pre-training dataset of short OTs is likely more efficient in reducing MAEs than adding more long OT data to the final training set, a good news from the perspective of computational cost. 

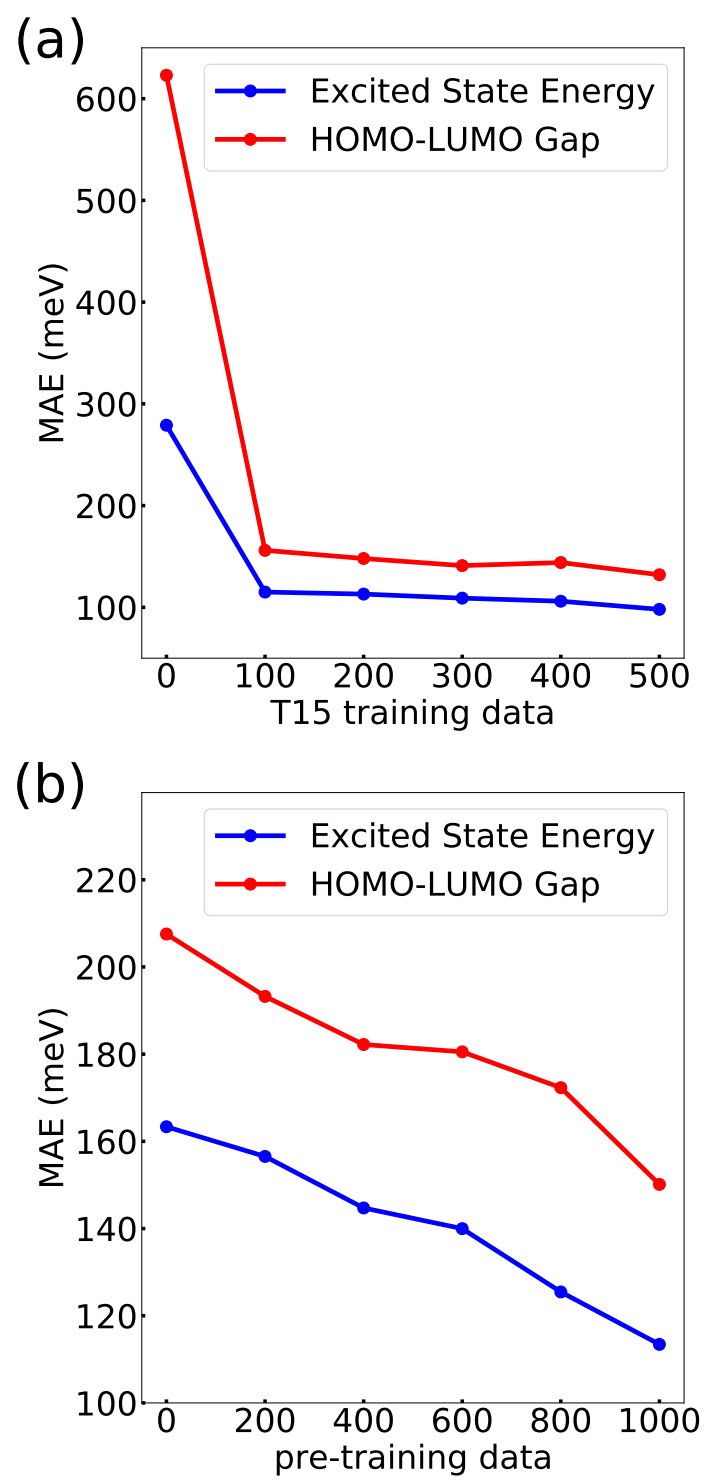

FIG. 3. MAEs of excited state energy (blue lines) and HOMO-LUMO gap (red lines) of $15 \mathrm{~T}$ as functions of $15 \mathrm{~T}$ training data (panel (a)) and as functions of pre-training data from each of the 2T-10T (panel (b)). 1000 training data from each of 2T-10T are used for panel (a), and $20015 \mathrm{~T}$ training data are used for panel (b).

\section{B. Justification for Transfer Learning}

To gain physical insights into the reason why pretraining ML models with data of short OTs could improve the predictions of long OTs, we analyse the electron density difference between the lowest-lying excited and ground states of $15 \mathrm{~T}$ molecule in Fig. 4. We consider electron density gain as electron and electron density depletion as hole upon electronic excitation. Fig. 4 (a) depicts the electron (blue) and hole (red) density of a typical $15 \mathrm{~T}$ configuration. It can be seen that both densities only occupy a small number of thiophene rings (a)

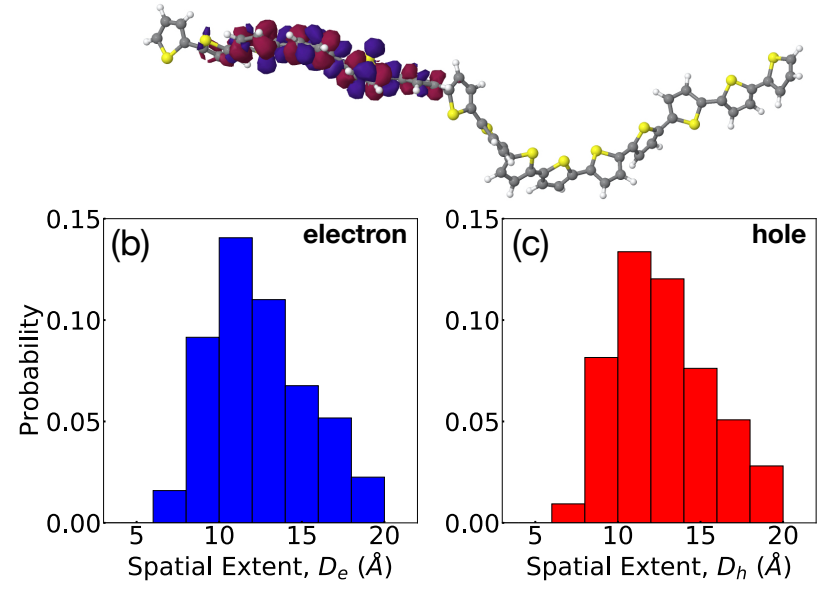

FIG. 4. (a) Electron density difference between the ground and first excited states of a representative 15T configuration. Electron density gain (loss), shown as the blue (red) 0.0005 iso-surface, is considered as electron (hole) density upon electronic excitation. (b) and (c) are the histograms of electron and hole spatial extent, respectively, generated from the electron density difference plots of $40015 \mathrm{~T}$ configurations. Note that the length of one thiophene ring is approximately $4 \AA$.

in the chain. This observation suggests that despite the long length of molecules like 15T, the electronic properties like excited-state energy are determined by a small fragment of the molecule. The limited delocalization of the electron/hole density is likely the result of the numerous kinks and distortions along the thiophene chain which prohibit the extension of electron and hole densities. This is consistent with many previous theoretical studies ${ }^{93-98}$ which found that conformational disorder due to thermal fluctuation or steric constraints (e.g., those caused by side chains) can localize the exciton in conjugated polymers.

To confirm the hypothesis that the electron and hole densities are relatively localized in most molecular configurations, we define electron/hole spatial extent following Ref. 99, which is given by

$$
D_{a}=2\left(\int\left(\vec{r}-\vec{r}_{a}\right)^{2} \rho_{a}(\vec{r}) d \vec{r} / \int \rho_{a}(\vec{r}) d \vec{r}\right)^{1 / 2}
$$

where $a=e, h$ for electron and hole, respectively, $\rho_{a}(\vec{r})$ is the electron/hole density, and the centroid of electron/hole density is given by

$$
\vec{r}_{a}=\int \vec{r} \rho_{a}(\vec{r}) d \vec{r} / \int \rho_{a}(\vec{r}) d \vec{r} .
$$

$D_{a}$ provides a rough measure of the delocalization extent of electron and hole densities, and the results for $40015 \mathrm{~T}$ configurations are shown as histograms in Fig. 4 (b) and (c). It can be seen that electron/hole spatial extent does not go beyond $20 \AA$, which is approximately the 
length of 5 thiophene rings (the length of one thiophene ring is about $4 \AA$ ). In fact, for most of the molecular configurations, electron/hole spatial extent is within the range of $10-15 \AA$. Some previous theoretical studies on $\mathrm{P}_{3} \mathrm{HT}^{95,98}$ showed that the electron or hole localization length is estimated to be around 5-7 thiophene rings at ambient temperature, slightly higher than our numbers for gas-phase $15 \mathrm{~T}$ at $1000 \mathrm{~K}$, although they adopted different measures to quantify delocalization. Additionally, we verify that the electron/hole spatial extent within a single 16-unit P3HT chain in its crystalline phase at 300 $\mathrm{K}$ (see Section III C for simulation setup) is also about 6 thiophene rings due to the distortions caused by thermal fluctuation and crystalline packing.

The relatively localized electron and hole densities indicate that ML models only need to learn the fragments of molecular chains with non-negligible electron/hole density, since the electronic properties are determined by these fragments which consists a smaller number of thiophene rings. Since the short OTs used in the pre-training phase may resemble these molecular fragments, they provide ML models with useful information in capturing the relation between atomic coordinates and quantum mechanical properties.

\section{Application to $\mathrm{P} 3 \mathrm{HT}$}

As an application of the transfer-learning model, we computed the excited state energies of P3HT in both its single-crystal phase and the dichloromethane solution. For both systems, classical MD simulations were performed for 16-unit regioregular P3HT using the OPLS/2005 force field, ${ }^{80}$ and the initial structure of P3HT was constructed using the geometric information in Ref. 100. For P3HT in dichloromethane, a single 16unit P3HT chain was immersed in a simulation box of 2876 dichloromethane molecules. Classical MD simulation was performed in the NPT ensemble at $300 \mathrm{~K}$ and $1 \mathrm{~atm}$ using the Martyna-Tobias-Klein scheme ${ }^{101}$ with a coupling constant of $2.0 \mathrm{ps}$, and after 100-ns equilibration, P3HT configurations were saved every 1 ps over a 10-ns production run. For the P3HT crystal, a total of 16 P3HT chains were initially stacked with their thiophene backbones perfectly aligned ${ }^{102}$ in the simulation box, whose dimensions match the estimated lattice constants in Ref. 103. NVT simulation was then performed using the Nosé-Hoover thermostat ${ }^{76,77}$ with a coupling constant of $2.0 \mathrm{ps}$. After 40-ns equilibration, P3HT configurations were saved every 10 ps over a 10 -ns production run. All other simulation details are the same as those for gas-phase OTs. 10000 P3HT configurations were harvested from the MD simulations for each system, and the hexyl side chains were replaced by $\mathrm{H}$ atoms for subsequent excited-state energy predictions since they are not involved in the $\pi$ conjugation.

We directly applied the transfer learning model for $16 \mathrm{~T}$ that was trained with the gas-phase data at $1000 \mathrm{~K}$ (i.e., (a)

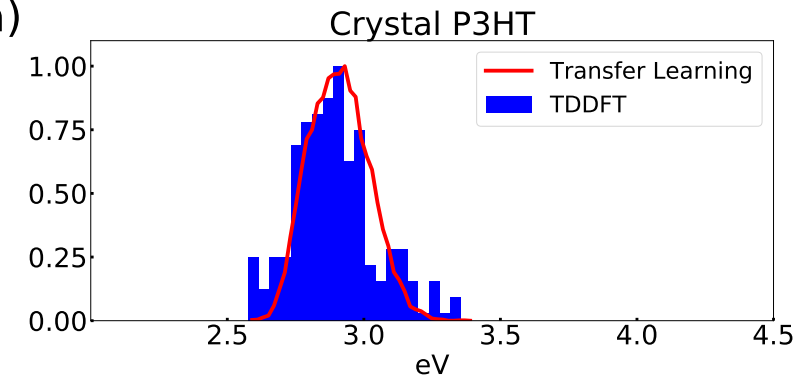

(b) Solution P3HT

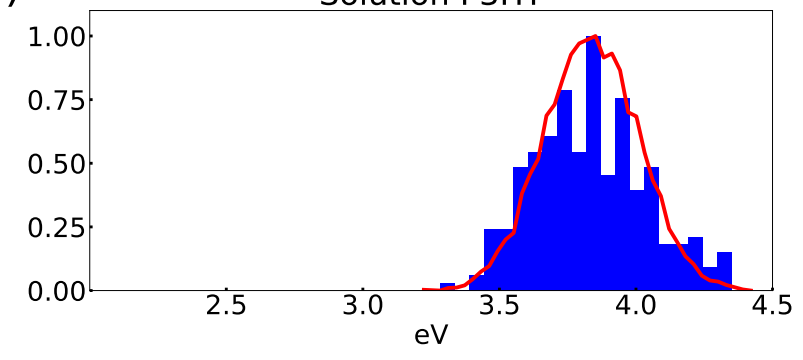

FIG. 5. The lowest excited-state energy distributions of P3HT in its single-crystal phase (top) and in dichloromethane solution (bottom), computed from the transfer learning model (red lines) and TDDFT calculations (blue bars). For each system, 10000 P3HT configurations were used in transferlearning prediction, whereas 400 configurations separated every $500 \mathrm{fs}$ were used for the TDDFT prediction. All the distributions are scaled to have the same peak height of 1 .

pre-trained on $2 \mathrm{~T}-10 \mathrm{~T}$ with 1000 data for each OT, and fined-tuned with 200 data for 16T) to obtain the excitedstate energies, and their distribution was then computed using

$$
f(E)=\left\langle\delta\left(E-\Omega_{i}\right)\right\rangle,
$$

where $\Omega_{i}$ is the lowest excited-state energy of the $i$ th (truncated) P3HT configuration, $\delta(x)$ is the Kronecker delta function, and the angular brackets indicate an ensemble average over 10000 configurations. Note that the excited-state energy distribution would be a good approximation to the UV-Vis absorption spectrum if excitonic couplings, motional narrowing, and non-Condon effects are negligible. ${ }^{104}$

The calculated lowest excited-state energy distributions from the transfer learning model are shown as red lines in Fig. 5, the distribution for the solution-phase spectrum is significantly blue-shifted and much broader compared to the that for crystal. To verify this trend, we randomly chose 400 configurations separated every 500 fs for each system to have their excited state energies computed directly from TDDFT, and the resulting distributions are shown as blue bars. It is evident that the transfer learning model faithfully reproduces the TDDFT results covering essentially the same energy ranges despite the fact that the configurations of P3HT in single crystal and solution could be very different from the gasphase $16 \mathrm{~T}$ configurations sampled in the model training. 
However, this might not be surprising since excitons are also relatively localized in $\mathrm{P} 3 \mathrm{HT}$ at ambient temperature due to conformational disorder as suggested by other theoretical studies. ${ }^{95,98}$ The blue-shifted and much broader distribution for the P3HT solution compared to that for the crystal from our simulation is also consistent with the experimental UV-Vis absorption spectra of P3HT solution and crystal, ${ }^{105,106}$ although the P3HT in the experiments is much longer potentially with substantial interchain and through-space intra-chain excitonic couplings. The successful application of the transfer learning model to P3HT shows the transferability of the trained model to different chemical environments, critical for its application to more relevant systems for optoelectronic devices, e.g., bulk heterojunctions for organic photovoltaics.

\section{CONCLUSION}

In this work, we have shown using OTs as the example that transfer learning with deep graph neural networks enables accurate predictions of electronic properties of long conjugated oligomers from those of short ones. This extends the conventional strategy of property extrapolation for optimized geometries to nonequilibrium configurations, and alleviates the data scarcity issue for big conjugated molecules. Prediction errors below $200 \mathrm{meV}$ in excited-state energy and HOMO-LUMO gap compared to DFT results can be achieved by transfer learning with even as few as 100 training data of target long OTs. We further show that the success of our transfer learning approach relies on the relative locality of low-lying electronic excitations in long conjugated OTs. The transfer learning model trained on gas-phase configurations shows good transferability in modeling the excited-state energy of P3HT in its single-crystal and solution phases. There remain challenges of applying transfer learning to model electronic properties of conjugated polymers, such as extension of the model to longer conjugated polymers and using higher-level quantum chemical methods than DFT for model training, and they will be interesting topics of future work.

\section{ACKNOWLEDGEMENTS}

L.S. acknowledges the support from the University of California Merced start-up funding. Q. L. acknowledges the support from the National Natural Science Foundation of China (Grant No. 61922073).

\footnotetext{
${ }^{1}$ L. Lu, T. Zheng, Q. Wu, A. M. Schneider, D. Zhao, and L. Yu, "Recent Advances in Bulk Heterojunction Polymer Solar Cells," Chemical Reviews 115, 12666-12731 (2015).

${ }^{2}$ M. Knaapila, ed., Conjugated Polymers and Oligomers: Structural and Soft Matter Aspects (World Scientific Publishing Co. Pte. Ltd., Singapore, 2017).

${ }^{3}$ J. R. Reynolds, B. C. Thompson, and T. A. Skotheim, eds., Conjugated Polymers: Perspective, Theory, and New Materials, 4th ed. (CRC Press/Taylor and Francis, Boca Raton, FL, 2019).
}

${ }^{4}$ B. Liu, ed., Conjugated Polymers for Biological and Biomedical Applications (WILEY-VCH Verlag GmbH \& Co. KGaA, Weinheim, Germany, 2018).

${ }^{5}$ H. Kuhn, "A Quantum-Mechanical Theory of Light Absorption of Organic Dyes and Similar Compounds," The Journal of Chemical Physics 17, 1198-1212 (1949).

${ }^{6}$ T. Izumi, S. Kobashi, K. Takimiya, Y. Aso, and T. Otsubo, "Synthesis and Spectroscopic Properties of a Series of $\beta$-Blocked Long Oligothiophenes up to the 96-mer: Revaluation of Effective Conjugation Length," Journal of the American Chemical Society 125, 5286-5287 (2003).

${ }^{7}$ G. R. Hutchison, Y.-J. Zhao, B. Delley, A. J. Freeman, M. A. Ratner, and T. J. Marks, "Electronic structure of conducting polymers: Limitations of oligomer extrapolation approximations and effects of heteroatoms," Physical Review B 68, 035204 (2003).

${ }^{8} \mathrm{C}$. Taliani and W. Gebauer, "Electronic Excited States of Conjugated Oligothiophenes," in Handbook of Oligo- and Polythiophenes (Wiley, Weinheim, Germany, 1998) pp. 361-404.

${ }^{9}$ S. S. Zade, N. Zamoshchik, and M. Bendikov, "From Short Conjugated Oligomers to Conjugated Polymers. Lessons from Studies on Long Conjugated Oligomers," Accounts of Chemical Research 44, 14-24 (2011).

${ }^{10} \mathrm{~J}$. Behler, "Neural network potential-energy surfaces in chemistry: a tool for large-scale simulations," Physical Chemistry Chemical Physics 13, 17930 (2011).

${ }^{11}$ J. Behler, "Constructing high-dimensional neural network potentials: A tutorial review," International Journal of Quantum Chemistry 115, 1032-1050 (2015).

${ }^{12} \mathrm{~J}$. Behler, "Perspective: Machine learning potentials for atomistic simulations," The Journal of Chemical Physics 145, 170901 (2016).

${ }^{13}$ P. O. Dral, "Quantum Chemistry in the Age of Machine Learning," Journal of Physical Chemistry Letters 11, 2336-2347 (2020).

${ }^{14}$ O. A. von Lilienfeld, K.-R. Müller, and A. Tkatchenko, "Exploring chemical compound space with quantum-based machine learning," Nature Reviews Chemistry 4, 347-358 (2020).

${ }^{15}$ F. Noé, A. Tkatchenko, K.-R. Müller, and C. Clementi, "Machine Learning for Molecular Simulation," Annual Review of Physical Chemistry 71, 361-390 (2020).

${ }^{16}$ R. Olivares-Amaya, C. Amador-Bedolla, J. Hachmann, S. Atahan-Evrenk, R. S. Sánchez-Carrera, L. Vogt, and A. Aspuru-Guzik, "Accelerated computational discovery of high-performance materials for organic photovoltaics by means of cheminformatics," Energy \& Environmental Science 4, 4849 (2011).

${ }^{17}$ R. Sajeev, R. S. Athira, M. Nufail, K. R. Jinu Raj, M. Rakhila, S. M. Nair, U. C. Abdul Jaleel, and A. T. Manuel, "Computational predictive models for organic semiconductors," Journal of Computational Electronics 12, 790-795 (2013).

${ }^{18}$ I. Y. Kanal, S. G. Owens, J. S. Bechtel, and G. R. Hutchison, "Efficient Computational Screening of Organic Polymer Photovoltaics," The Journal of Physical Chemistry Letters 4, 16131623 (2013).

${ }^{19}$ Y. Shu and B. G. Levine, "Simulated evolution of fluorophores for light emitting diodes," The Journal of Chemical Physics 142, 104104 (2015).

${ }^{20}$ H. Li, Z. Zhong, L. Li, R. Gao, J. Cui, T. Gao, L. H. Hu, Y. Lu, Z.-M. Su, and H. Li, "A cascaded QSAR model for efficient prediction of overall power conversion efficiency of all-organic dye-sensitized solar cells," Journal of Computational Chemistry 36, 1036-1046 (2015).

${ }^{21}$ E. O. Pyzer-Knapp, G. N. Simm, and A. Aspuru Guzik, "A Bayesian approach to calibrating high-throughput virtual screening results and application to organic photovoltaic materials," Materials Horizons 3, 226-233 (2016).

${ }^{22}$ F. Pereira, K. Xiao, D. A. R. S. Latino, C. Wu, Q. Zhang, and J. Aires-de Sousa, "Machine Learning Methods to Predict Density Functional Theory B3LYP Energies of HOMO and LUMO 
Orbitals," Journal of Chemical Information and Modeling 57, 11-21 (2017).

${ }^{23}$ R. Gómez-Bombarelli, J. Aguilera-Iparraguirre, T. D. Hirzel, D. Duvenaud, D. Maclaurin, M. A. Blood-Forsythe, H. S. Chae, M. Einzinger, D.-G. G. Ha, T. Wu, G. Markopoulos, S. Jeon, H. Kang, H. Miyazaki, M. Numata, S. Kim, W. Huang, S. I. Hong, M. Baldo, R. P. Adams, and A. Aspuru-Guzik, "Design of efficient molecular organic light-emitting diodes by a highthroughput virtual screening and experimental approach," Nature Materials 15, 1120-1127 (2016).

${ }^{24}$ S.-Y. Lu, S. Mukhopadhyay, R. Froese, and P. M. Zimmerman, "Virtual Screening of Hole Transport, Electron Transport, and Host Layers for Effective OLED Design," Journal of Chemical Information and Modeling 58, 2440-2449 (2018).

${ }^{25}$ F. Musil, S. De, J. Yang, J. E. Campbell, G. M. Day, and M. Ceriotti, "Machine learning for the structure-energy-property landscapes of molecular crystals," Chemical Science 9, 12891300 (2018).

${ }^{26}$ N. Mahapatra, A. Ben-Cohen, Y. Vaknin, A. Henning, J. Hayon, K. Shimanovich, H. Greenspan, and Y. Rosenwaks, "Electrostatic Selectivity of Volatile Organic Compounds Using Electrostatically Formed Nanowire Sensor," ACS Sensors 3, 709-715 (2018).

${ }^{27}$ S. Nagasawa, E. Al-Naamani, and A. Saeki, "Computer-Aided Screening of Conjugated Polymers for Organic Solar Cell: Classification by Random Forest," The Journal of Physical Chemistry Letters 9, 2639-2646 (2018).

${ }^{28}$ P. B. Jørgensen, M. Mesta, S. Shil, J. M. García Lastra, K. W. Jacobsen, K. S. Thygesen, and M. N. Schmidt, "Machine learning-based screening of complex molecules for polymer solar cells," The Journal of Chemical Physics 148, 241735 (2018).

${ }^{29}$ M. A. B. Janai, K. L. Woon, and C. S. Chan, "Design of efficient blue phosphorescent bottom emitting light emitting diodes by machine learning approach," Organic Electronics 63, 257-266 (2018).

${ }^{30}$ H. Sahu, W. Rao, A. Troisi, and H. Ma, "Toward Predicting Efficiency of Organic Solar Cells via Machine Learning and Improved Descriptors," Advanced Energy Materials 8, 1801032 (2018).

${ }^{31}$ D. Padula and A. Troisi, "Concurrent Optimization of Organic Donor-Acceptor Pairs through Machine Learning," Advanced Energy Materials 9, 1902463 (2019).

${ }^{32}$ D. Padula, J. D. Simpson, and A. Troisi, "Combining electronic and structural features in machine learning models to predict organic solar cells properties," Materials Horizons 6, 343-349 (2019).

${ }^{33}$ M. Lee, "Insights from Machine Learning Techniques for Predicting the Efficiency of Fullerene Derivatives-Based Ternary Organic Solar Cells at Ternary Blend Design," Advanced Energy Materials 9, 1900891 (2019).

${ }^{34}$ P. C. St. John, C. Phillips, T. W. Kemper, A. N. Wilson, Y. Guan, M. F. Crowley, M. R. Nimlos, and R. E. Larsen, "Message-passing neural networks for high-throughput polymer screening," The Journal of Chemical Physics 150, 234111 (2019), arXiv:1807.10363.

${ }^{35}$ S. Atahan-Evrenk and F. B. Atalay, "Prediction of Intramolecular Reorganization Energy Using Machine Learning," The Journal of Physical Chemistry A 123, 7855-7863 (2019).

${ }^{36}$ L. Bian, D. C. Sorescu, L. Chen, D. L. White, S. C. Burkert, Y. Khalifa, Z. Zhang, E. Sejdic, and A. Star, "MachineLearning Identification of the Sensing Descriptors Relevant in Molecular Interactions with Metal Nanoparticle-Decorated Nanotube Field-Effect Transistors," ACS Applied Materials \& Interfaces 11, 1219-1227 (2019).

${ }^{37}$ J. Lederer, W. Kaiser, A. Mattoni, and A. Gagliardi, "Machine Learning-Based Charge Transport Computation for Pentacene," Advanced Theory and Simulations 2, 1800136 (2019).

${ }^{38}$ L. M. Roch, S. K. Saikin, F. Häse, P. Friederich, R. H. Goldsmith, S. León, and A. Aspuru-Guzik, "From Absorption Spectra to Charge Transfer in Nanoaggregates of Oligomers with
Machine Learning," ACS Nano 14, 6589-6598 (2020).

${ }^{39}$ L. Simine, T. C. Allen, and P. J. Rossky, "Predicting optical spectra for optoelectronic polymers using coarse-grained models and recurrent neural networks," Proceedings of the National Academy of Sciences 117, 13945-13948 (2020).

${ }^{40}$ C. Lu, Q. Liu, Q. Sun, C.-Y. Hsieh, S. Zhang, L. Shi, and C.-K. Lee, "Deep Learning for Optoelectronic Properties of Organic Semiconductors," The Journal of Physical Chemistry C 124, 7048-7060 (2020).

${ }^{41}$ A. Farahvash, C.-K. Lee, Q. Sun, L. Shi, and A. P. Willard, "Machine learning Frenkel Hamiltonian parameters to accelerate simulations of exciton dynamics," The Journal of Chemical Physics 153, 074111 (2020).

${ }^{42}$ K. T. Schütt, H. E. Sauceda, P.-J. Kindermans, A. Tkatchenko, and K.-R. Müller, "SchNet - A deep learning architecture for molecules and materials," The Journal of Chemical Physics 148, $241722(2018)$.

${ }^{43}$ J. Gilmer, S. S. Schoenholz, P. F. Riley, O. Vinyals, and G. E. Dahl, "Neural message passing for quantum chemistry," in Proceedings of the 34th International Conference on Machine Learning-Volume 70 (JMLR. org, 2017) pp. 1263-1272.

${ }^{44}$ R. Ramakrishnan, P. O. Dral, M. Rupp, and O. A. von Lilienfeld, "Quantum chemistry structures and properties of 134 kilo molecules," Scientific Data 1, 140022 (2014).

${ }^{45}$ D. K. Duvenaud, D. Maclaurin, J. Iparraguirre, R. Bombarell, T. Hirzel, A. Aspuru-Guzik, and R. P. Adams, "Convolutional Networks on Graphs for Learning Molecular Fingerprints," in Advances in Neural Information Processing Systems (2015) pp. $2224-2232$.

${ }^{46}$ K. T. Schütt, F. Arbabzadah, S. Chmiela, K. R. Müller, and A. Tkatchenko, "Quantum-chemical insights from deep tensor neural networks," Nature Communications 8, 13890 (2017), arXiv:arXiv: $1609.08259 \mathrm{v} 4$

${ }^{47}$ Z. Wu, B. Ramsundar, E. N. Feinberg, J. Gomes, C. Geniesse, A. S. Pappu, K. Leswing, and V. Pande, "MoleculeNet: a benchmark for molecular machine learning," Chemical Science 9, 513-530 (2018).

${ }^{48}$ C. Lu, Q. Liu, C. Wang, Z. Huang, P. Lin, and L. He, "Molecular Property Prediction: A Multilevel Quantum Interactions Modeling Perspective," Proceedings of the AAAI Conference on Artificial Intelligence 33, 1052-1060 (2019).

${ }^{49}$ K. T. Schütt, M. Gastegger, A. Tkatchenko, K.-R. Müller, and R. J. Maurer, "Unifying machine learning and quantum chemistry with a deep neural network for molecular wavefunctions," Nature Communications 10, 5024 (2019), arXiv:1906.10033.

${ }^{50}$ G. Chen, P. Chen, C.-Y. Hsieh, C.-K. Lee, B. Liao, R. Liao, W. Liu, J. Qiu, Q. Sun, J. Tang, R. Zemel, and S. Zhang, "Alchemy: A Quantum Chemistry Dataset for Benchmarking AI Models," , arXiv:1906.09427 (2019), arXiv:1906.09427.

${ }^{51}$ O. T. Unke and M. Meuwly, "PhysNet: A Neural Network for Predicting Energies, Forces, Dipole Moments, and Partial Charges," Journal of Chemical Theory and Computation 15, 3678-3693 (2019).

${ }^{52}$ J. Klicpera, J. Groß, and S. Günnemann, "Directional Message Passing for Molecular Graphs," , arXiv:2003.03123 (2020), arXiv:2003.03123.

${ }^{53}$ Z. Liu, L. Lin, Q. Jia, Z. Cheng, Y. Jiang, Y. Guo, and J. Ma, "Transferable Multi-level Attention Neural Network for Accurate Prediction of Quantum Chemistry Properties via Multitask Learning," , chemRxiv:12588170 (2020).

${ }^{54}$ Z. Qiao, M. Welborn, A. Anandkumar, F. R. Manby, and T. F. Miller, "OrbNet: Deep learning for quantum chemistry using symmetry-adapted atomic-orbital features," The Journal of Chemical Physics 153, 124111 (2020), arXiv:2007.08026.

${ }^{55}$ Z. Hao, C. Lu, Z. Hu, H. Wang, Z. Huang, Q. Liu, E. Chen, and C. Lee, "ASGN: An Active Semi-supervised Graph Neural Network for Molecular Property Prediction," , arXiv:2007.03196 (2020), arXiv:2007.03196.

${ }^{56}$ S. J. Pan and Q. Yang, "A Survey on Transfer Learning," IEEE Transactions on Knowledge and Data Engineering 22, 1345- 
1359 (2010).

${ }^{57}$ K. Weiss, T. M. Khoshgoftaar, and D. Wang, "A survey of transfer learning," Journal of Big Data 3, 9 (2016).

${ }^{58}$ J. S. Smith, B. T. Nebgen, R. Zubatyuk, N. Lubbers, C. Devereux, K. Barros, S. Tretiak, O. Isayev, and A. E. Roitberg, "Approaching coupled cluster accuracy with a generalpurpose neural network potential through transfer learning," Nature Communications 10, 2903 (2019).

${ }^{59}$ N. Dandu, L. Ward, R. S. Assary, P. C. Redfern, B. Narayanan, I. T. Foster, and L. A. Curtiss, "Quantum-Chemically Informed Machine Learning: Prediction of Energies of Organic Molecules with 10 to 14 Non-hydrogen Atoms," The Journal of Physical Chemistry A 124, 5804-5811 (2020).

${ }^{60}$ H. Yamada, C. Liu, S. Wu, Y. Koyama, S. Ju, J. Shiomi, J. Morikawa, and R. Yoshida, "Predicting Materials Properties with Little Data Using Shotgun Transfer Learning," ACS Central Science 5, 1717-1730 (2019).

${ }^{61} \mathrm{X} . \mathrm{Li}$ and $\mathrm{D}$. Fourches, "Inductive transfer learning for molecular activity prediction: Next-Gen QSAR Models with MolPMoFiT," Journal of Cheminformatics 12, 1-15 (2020).

${ }^{62}$ R. S. Simões, V. G. Maltarollo, P. R. Oliveira, and K. M. Honorio, "Transfer and Multi-task Learning in QSAR Modeling: Advances and Challenges," Frontiers in Pharmacology 9, 74 (2018).

${ }^{63}$ A. Paul, D. Jha, R. Al-Bahrani, W. K. Liao, A. Choudhary, and A. Agrawal, "Transfer Learning Using Ensemble Neural Networks for Organic Solar Cell Screening," Proceedings of the International Joint Conference on Neural Networks 2019-July, 1-8 (2019), arXiv:1903.03178.

${ }^{64}$ E. D. Cubuk, A. D. Sendek, and E. J. Reed, "Screening billions of candidates for solid lithium-ion conductors: A transfer learning approach for small data," The Journal of Chemical Physics 150, 214701 (2019).

${ }^{65}$ N. C. Iovanac and B. M. Savoie, "Improved Chemical Prediction from Scarce Data Sets via Latent Space Enrichment," Journal of Physical Chemistry A 123, 4295-4302 (2019).

${ }^{66}$ N. C. Iovanac and B. M. Savoie, "Simpler is Better: How Linear Prediction Tasks Improve Transfer Learning in Chemical Autoencoders," Journal of Physical Chemistry A 124, 3679-3685 (2020).

${ }^{67}$ D. Fichou, Handbook of Oligo- and Polythiophenes, edited by D. Fichou (Wiley, Weinheim, Germany, 1998).

${ }^{68}$ I. F. Perepichka and D. F. Perepichka, Handbook of ThiopheneBased Materials: Applications in Organic Electronics and Photonics, edited by I. F. Perepichka and D. F. Perepichka (John Wiley \& Sons, Ltd, Chichester, UK, 2009).

${ }^{69}$ E. Havinga, I. Rotte, E. Meijer, W. Hoeve, and H. Wynberg, "Spectra and electrical properties of soluble partially alkylsubstituted oligomers of thiophene up to 11 rings," Synthetic Metals 41, 473-478 (1991).

${ }^{70}$ W. Ten Hoeve, H. Wynberg, E. E. Havinga, and E. W. Meijer, "Substituted 2,2':5',2":5",2"':5", 2 " ": 5 ", , 2 "'",

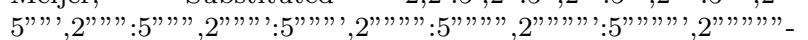
undecithiophenes, the longest characterized oligothiophenes," Journal of the American Chemical Society 113, 5887-5889 (1991).

${ }^{71}$ D. Fichou, G. Horowitz, B. Xu, and F. Garnier, "Low temperature optical absorption of polycrystalline thin films of $\alpha$ quaterthiophene, $\alpha$-sexithiophene and $\alpha$-octithiophene, three model oligomers of polythiophene," Synthetic Metals 48, 167179 (1992).

${ }^{72}$ H. Meier, U. Stalmach, and H. Kolshorn, "Effective conjugation length and UV/vis spectra of oligomers," Acta. Polymer 48, 379-384 (1997).

${ }^{73}$ A. Yang, M. Kuroda, Y. Shiraishi, and T. Kobayashi, "ChainLength Dependent Stationary and Time-Resolved Spectra of $\alpha$ Oligothiophenes," The Journal of Physical Chemistry B 102, 3706-3711 (1998).

${ }^{74}$ J. S. de Melo, L. M. Silva, L. G. Arnaut, and R. S. Becker, "Singlet and triplet energies of $\alpha$-oligothiophenes: A spectro- scopic, theoretical, and photoacoustic study: Extrapolation to polythiophene," The Journal of Chemical Physics 111, 54275433 (1999).

${ }^{75}$ E. Fabiano, F. D. Sala, R. Cingolani, M. Weimer, and A. Görling, "Theoretical Study of Singlet and Triplet Excitation Energies in Oligothiophenes," The Journal of Physical Chemistry A 109, 3078-3085 (2005).

${ }^{76} \mathrm{~S}$. Nosé, "A molecular dynamics method for simulations in the canonical ensemble," Molecular Physics 52, 255-268 (1984).

${ }^{77}$ W. G. Hoover, "Canonical dynamics: Equilibrium phase-space distributions," Physical Review A 31, 1695-1697 (1985).

${ }^{78}$ T. Darden, D. York, and L. Pedersen, "Particle mesh Ewald: An N. $\log (\mathrm{N})$ method for Ewald sums in large systems," The Journal of Chemical Physics 98, 10089-10092 (1993).

${ }^{79}$ U. Essmann, L. Perera, M. L. Berkowitz, T. Darden, H. Lee, and L. G. Pedersen, "A smooth particle mesh Ewald method," The Journal of Chemical Physics 103, 8577-8593 (1995).

${ }^{80}$ J. L. Banks, H. S. Beard, Y. Cao, A. E. Cho, W. Damm, R. Farid, A. K. Felts, T. a. Halgren, D. T. Mainz, J. R. Maple, R. Murphy, D. M. Philipp, M. P. Repasky, L. Y. Zhang, B. J. Berne, R. a. Friesner, E. Gallicchio, and R. M. Levy, "Integrated Modeling Program, Applied Chemical Theory (IMPACT)," Journal of Computational Chemistry 26, 1752-1780 (2005).

${ }^{81}$ K. J. Bowers, F. D. Sacerdoti, J. K. Salmon, Y. Shan, D. E. Shaw, E. Chow, H. Xu, R. O. Dror, M. P. Eastwood, B. A. Gregersen, J. L. Klepeis, I. Kolossvary, and M. A. Moraes, "Scalable algorithms for molecular dynamics simulations on commodity clusters," in Proceedings of the 2006 ACM/IEEE conference on Supercomputing - SC '06 (ACM Press, New York, New York, USA, 2006) p. 84.

${ }^{82}$ K. H. Dubay, M. L. Hall, T. F. Hughes, C. Wu, D. R. Reichman, and R. a. Friesner, "Accurate force field development for modeling conjugated polymers," Journal of Chemical Theory and Computation 8, 4556-4569 (2012).

${ }^{83}$ Q. Sun, T. C. Berkelbach, N. S. Blunt, G. H. Booth, S. Guo, Z. Li, J. Liu, J. D. McClain, E. R. Sayfutyarova, S. Sharma, S. Wouters, and G. K.-L. Chan, "PySCF: the Python-based simulations of chemistry framework," Wiley Interdisciplinary Reviews: Computational Molecular Science 8, e1340 (2018).

${ }^{84}$ M. Rupp, A. Tkatchenko, K.-R. Müller, and O. A. von Lilienfeld, "Fast and Accurate Modeling of Molecular Atomization Energies with Machine Learning," Physical Review Letters 108, 058301 (2012).

${ }^{85}$ K. Hansen, G. Montavon, F. Biegler, S. Fazli, M. Rupp, M. Scheffler, O. A. von Lilienfeld, A. Tkatchenko, and K.-R. Müller, "Assessment and Validation of Machine Learning Methods for Predicting Molecular Atomization Energies," Journal of Chemical Theory and Computation 9, 3404-3419 (2013).

${ }^{86} \mathrm{~K}$. Hansen, F. Biegler, R. Ramakrishnan, W. Pronobis, O. A. von Lilienfeld, K.-R. Müller, and A. Tkatchenko, "Machine Learning Predictions of Molecular Properties: Accurate ManyBody Potentials and Nonlocality in Chemical Space," The Journal of Physical Chemistry Letters 6, 2326-2331 (2015).

${ }^{87}$ A. P. Bartók, R. Kondor, and G. Csányi, "On representing chemical environments," Physical Review B 87, 184115 (2013).

${ }^{88} \mathrm{~J}$. Behler, "Atom-centered symmetry functions for constructing high-dimensional neural network potentials," The Journal of Chemical Physics 134, 074106 (2011).

${ }^{89}$ D. P. Kingma and J. Ba, "Adam: A method for stochastic optimization," in International Conference on Learning Representations (ICLR) (2015).

${ }^{90}$ P. Adam, C. Soumith, C. Gregory, Y. Edward, D. Zachary, L. Zeming, D. Alban, A. Luca, and L. Adam, "Automatic differentiation in pytorch," in Proceedings of Neural Information Processing Systems (2017).

${ }^{91}$ M. Wang, L. Yu, D. Zheng, Q. Gan, Y. Gai, Z. Ye, M. Li, J. Zhou, Q. Huang, C. Ma, Z. Huang, Q. Guo, H. Zhang, H. Lin, J. Zhao, J. Li, A. J. Smola, and Z. Zhang, "Deep Graph Library: Towards Efficient and Scalable Deep Learning 
on Graphs," ICLR Workshop on Representation Learning on Graphs and Manifolds (2019).

92 "Source Codes,".

${ }^{93} \mathrm{~W}$. Barford and D. Trembath, "Exciton localization in polymers with static disorder," Physical Review B 80, 165418 (2009).

${ }^{94}$ W. Barford, D. G. Lidzey, D. V. Makhov, and A. J. H. Meijer, "Exciton localization in disordered poly(3-hexylthiophene)," The Journal of Chemical Physics 133, 044504 (2010).

${ }^{95}$ N. Vukmirovi $\backslash\{$ c $\}$ and L.-W. Wang, "Density of States and Wave Function Localization in Disordered Conjugated Polymers: A Large Scale Computational Study," The Journal of Physical Chemistry B 115, 1792-1797 (2011).

${ }^{96} \mathrm{H}$. Ma, T. Qin, and A. Troisi, "Electronic Excited States in Amorphous MEH-PPV Polymers from Large-Scale First Principles Calculations," Journal of Chemical Theory and Computation 10, 1272-1282 (2014).

${ }^{97}$ M. C. da Silva, S. d. M. Cassemiro, A. M. Machado, J. C. F. Alves, S. L. Nogueira, T. Jarrosson, F. Serein-Spirau, L. Akcelrud, J. R. Tozoni, R. A. Silva, J. A. Freire, and A. Marletta, "Conjugation Length Distribution in Poly( $\mathrm{p}$ phenylenevinylene) (PPV) Films," The Journal of Physical Chemistry A 120, 9702-9706 (2016).

${ }^{98}$ L. Simine and P. J. Rossky, "Relating Chromophoric and Structural Disorder in Conjugated Polymers," The Journal of Physical Chemistry Letters 8, 1752-1756 (2017).

${ }^{99}$ T. Le Bahers, C. Adamo, and I. Ciofini, "A qualitative index of spatial extent in charge-transfer excitations," Journal of Chem- ical Theory and Computation 7, 2498-2506 (2011).

${ }^{100}$ R. Alessandri, J. J. Uusitalo, A. H. de Vries, R. W. A. Havenith, and S. J. Marrink, "Bulk Heterojunction Morphologies with Atomistic Resolution from Coarse-Grain Solvent Evaporation Simulations," Journal of the American Chemical Society 139, 3697-3705 (2017).

${ }^{101}$ G. J. Martyna, D. J. Tobias, and M. L. Klein, "Constant pressure molecular dynamics algorithms," The Journal of Chemical Physics 101, 4177 (1994).

${ }^{102}$ R. D. McCullough, S. Tristram-Nagle, S. P. Williams, R. D. Lowe, and M. Jayaraman, "Self-orienting head-to-tail poly(3alkylthiophenes): new insights on structure-property relationships in conducting polymers," Journal of the American Chemical Society 115, 4910-4911 (1993).

${ }^{103}$ T. J. Prosa, M. J. Winokur, J. Moulton, P. Smith, and A. J. Heeger, "X-ray structural studies of poly(3-alkylthiophenes): an example of an inverse comb," Macromolecules 25, 4364-4372 (1992).

${ }^{104}$ D. A. McQuarrie, Statistical Mechanics (Harper and Row, New York, 1976).

${ }^{105}$ S. Hotta and K. Ito, "Electronic Properties of Polythiophenes," in Handbook of Oligo- and Polythiophenes (Wiley, 1998) pp. 45-87.

${ }^{106}$ K. Rahimi, I. Botiz, J. O. Agumba, S. Motamen, N. Stingelin, and G. Reiter, "Light absorption of poly(3-hexylthiophene) single crystals," RSC Adv. 4, 11121-11123 (2014). 